\title{
Apolipoprotein E phenotype, cholesterol and breast and prostate cancer
}

\begin{abstract}
Mari Niemi, Kari Kervinen, Heikki Kiviniemi, Olavi Lukkarinen, Atte-Pekka Kyllönen, Meeri Apaja-Sarkkinen, Markku J Savolainen, Matti I Kairaluoma, Y Antero Kesäniemi
\end{abstract}

Although many studies have shown an increased risk of death from cancer in subjects with low plasma cholesterol concentrations, the underlying mechanism between low blood cholesterol and cancer is unclear. Apolipoprotein $\mathrm{E}$ is a central regulatory protein in cholesterol metabolism, and recent studies have shown that apolipoprotein $\mathrm{E}$ is a potent inhibitor of angiogenesis and tumour cell growth. ${ }^{1}$ Apolipoprotein $\mathrm{E}$ has three common isoforms, E2, E3, and E4, which are coded by the alleles e2, e 3 and e4, respectively. The e4 allele is associated with high serum total and low density lipoprotein cholesterol concentrations. ${ }^{2}$ Recently, it was suggested that the apolipoprotein $\mathrm{E}$ e4 allele may protect from carcinoma and adenoma of the colon. ${ }^{3}$ We hypothesised that the apolipoprotein E polymorphism could also be associated with other cancer types. The main question of this study was whether the apolipoprotein $\mathrm{E}$ allele distribution is different in patients with cancer of the breast or prostate compared with patients with benign breast disease (BBD) or benign prostate hyperplasia $(\mathrm{BPH})$ and a random population. The possible abnormalities in serum cholesterol and its fractions in patients with cancer of the breast or prostate in comparison with non-cancer patients were also investigated.

Internal Medicine, University of Oulu,

Finland

M Niemi

K Kervinen

M J Savolainen

Y Antero Kesäniemi

Department of

Surgery, University of

Oulu

H Kiviniemi

O Lukkarinen

M I Kairaluoma

Biocenter Oulu,

University of Oulu

$\mathrm{M}$ Niemi

K Kervinen

M J Savolainen

Y Antero Kesäniemi

Department of

Pathology, Oulu

University Hospital

A-P Kyllönen

M Apaja-Sarkkinen

Correspondence to: Professor Kesäniemi, Department of Internal

Medicine, University of Oulu, Kajaanintie 50,

FIN-90220 Oulu, Finland

(antero.kesaniemi@oulu.fi)

Accepted for publication 20 June 2000 tion of Oulu. All the patients gave informed men with benign prostate hyperplasia and men with prostate cancer benign prostate hyperplasia.

\section{Methods}

undergoing surgery for breast carcinoma, 299 undergoing tretment for prostate cancer, patient and control groups with a populationbased cohort in northern Finland, 267 women and 259 men aged 54-61 years were randomly selected by age stratification from the social insurance register covering the whole popula- consent for the investigations, which were approved by the Ethical Committee of the University of Oulu.

Venous blood samples were drawn into EDTA tubes after an overnight fast. The plasma low density and high density lipoprotein cholesterol concentrations were estimated as described in the Lipid Research Clinics Manual of Laboratory Operations. ${ }^{4}$ Cholesterol and triglycerides were analysed enzymatically with kits of Boehringer Diagnostica, Mannheim GmbH, Germany. The apolipoprotein $\mathrm{E}$ phenotype was determined from the plasma with the isoelectric focusing and immunoblotting techniques.

The results were expressed as means (95\% confidence intervals). The differences in the means were calculated with the analysis of variance. For triglycerides, which did not follow a normal distribution, the analysis was performed after logarithmic transformation. The distribution of allele frequencies was assessed with the $\chi^{2}$ test.

\section{Results}

The allelic frequencies of apolipoprotein E did not differ between the patients with breast cancer (with frequencies of $0.040,0.784$, and 0.175 for the e2, e3 and e4 alleles, respectively), the patients with $\mathrm{BBD},(0.036,0.780$ and 0.182 ) and the control women (0.054, 0.764 and 0.182 ). Also, the apolipoprotein $\mathrm{E}$ allelic frequencies were equal in the men with prostate cancer (0.031, 0.808 and 0.165), those with $\mathrm{BPH}(0.032,0.838$ and 0.129$)$ and the control men (0.037, 0.760 and 0.203).

Plasma concentrations of cholesterol and its fractions in the cancer and non-cancer patients are shown in table 1 . After the age adjustment there were no differences between the women with breast cancer and the women with $\mathrm{BBD}$ (table 1). The men with prostate cancer had significantly higher mean values for total, LDL and HDL cholesterol than the men with $\mathrm{BPH}$ (table 1). There were no significant

Table 1 Clinical characteristics and plasma lipids of women with benign breast disease and women with breast cancer and

\begin{tabular}{|c|c|c|c|c|}
\hline & Benign breast disease & Breast cancer & $\begin{array}{l}\text { Benign prostate } \\
\text { hyperplasia }\end{array}$ & Prostate cancer \\
\hline Number & 299 & 211 & 201 & 130 \\
\hline Age (y) & $43.3(41.9,44.7)$ & $55.2(53.4,57.0)$ & $69.7(68.6,70.7)$ & $68.8(67.2,70.2)$ \\
\hline $\mathrm{BMI}\left(\mathrm{kg} / \mathrm{m}^{2}\right)$ & $25.2(24.3,26.3)$ & $25.9(24.3,26.3)$ & $26.5(25.9,26.9)$ & $26.4(25.8,27.1)$ \\
\hline Plasma cholesterol $(\mathrm{mmol} / \mathrm{l})$ & $5.2(5.1,5.38)$ & $5.1(5.0,5.3)$ & $4.9(4.8,5.0)$ & $5.4(5 \cdot 2,5.6)^{\star}$ \\
\hline LDL cholesterol $(\mathrm{mmol} / \mathrm{l})$ & $2.8(2.7,2.9)$ & $2.7(2.6,2.9)$ & $3.0(2.9,3.1)$ & $3.3(3.1,3.5) \dagger$ \\
\hline HDL cholesterol (mmol/1) & $1.6(1.5,1.7)$ & $1.6(1.5,1.7)$ & $1.2(1.2,1.3)$ & $1.5(1.3,1.5)^{\star}$ \\
\hline Triglycerides $(\mathrm{mmol} / \mathrm{l})$ & $1.2(1.2,1.3)$ & $1.2(1.1,1.3)$ & $1.4(1.3,1.5)$ & $1.5(1.4,1.6)$ \\
\hline
\end{tabular}

The values are presented as means ( $95 \%$ confidence intervals). Logarithmic transformation was done for the analyses of total triglycerides. ${ }^{\star} \mathrm{p}<0.0001$ for the difference compared with benign prostate hyperplasia. $t \mathrm{p}<0.007$ for the difference compared with 
differences in triglyceride levels between the patients with prostate cancer and $\mathrm{BPH}$. The men with prostate cancer had significantly higher plasma total, LDL and HDL cholesterol in the apolipoprotein E groups E3 and $\mathrm{E} 4$, and lower serum total, LDL and HDL cholesterol concentrations in the apolipoprotein $\mathrm{E} 2$ group when compared with $\mathrm{BPH}$.

\section{Comment}

This study did not show any evidence that the genetically determined apolipoprotein E phenotype would be associated with breast cancer in women or with prostate cancer in men. High plasma cholesterol, particularly LDL and HDL cholesterol concentrations were observed in the patients with prostate cancer.
The skilful technical assistance of Ms Saija Kortetjärvi, Ms Eila Saarikoski, Ms Liisa Mannermaa and Ms Leena Ukkola is greatly appreciated.

Funding: this work was supported by the Medical Council of the Academy of Finland and the Cancer Society of Northern Finland.

Conflicts of interest: none.

1 Vogel T, Guo NH, Guy R, et al. Apolipoprotein E: a potent inhibitor of endothelial and tumor cell proliferation. $\mathcal{F}$ Cell Biochem 1994;54:299-308.

2 Utermann G. Apolipoprotein $\mathrm{E}$ in health and disease. $\mathrm{Am}$ Heart f 1987;113:433-40.

3 Kervinen K, Södervik H, Mäkelä J, et al. Is the development Kervinen K, Södervik $\mathrm{H}$, Mäkelä J, et al. Is the development
of adenoma and carcinoma in proximal colon related to apolipoprotein E phenotype? Gastroenterology 1996;110: 1785-90

4 Lipid Research Clinics Program. Manual of laboratory operations. Lipid and lipoprotein analysis. Bethesda, MD: DHEW publications, National Institutes of Health, 1974 